\title{
Study of carbon nanotube-rich impedimetric recognition electrode for ultra-low determination of polycyclic aromatic hydrocarbons in water
}

\author{
Jose Muñoz, ${ }^{\mathrm{a}}$ Cristina Navarro-Senent, ${ }^{\mathrm{a}}$ Nuria Crivillers ${ }^{\mathrm{a}, \mathrm{b}}$ and Marta Mas-Torrent ${ }^{\mathrm{a}, \mathrm{b},{ }^{*}}$ \\ ${ }^{a}$ Institut de Ciència de Materials de Barcelona (ICMAB-CSIC), Campus de la UAB, 08193, Bellaterra, Spain \\ ${ }^{b}$ Networking Research Center on Bioengineering Biomaterials and Nanomedicine (CIBER-BBN), Campus de la UAB, 08193 \\ Bellaterra, Spain \\ *Corresponding author: mmas@icmab.es
}

\section{ABSTRACT}

Carbon nanotubes (CNTs) have been studied as an electrochemical recognition element for the impedimetric determination of priority polycyclic aromatic hydrocarbons (PAHs) in water, using hexocyanoferrate as a redox probe. For this goal, an Indium Tin Oxide (ITO) electrode functionalized with a silane-based self-assembled monolayer carrying CNTs has been engineered. The electroanalytical method, which is similar to an antibody-antigen assay, is straightforward and focused on exploiting the high CNTPAH affinity obtained via $\pi$-interactions. After optimizing the experimental conditions, the resulting CNT-based impedimetric recognition platform exhibits ultra-low detection limits $\left(1.75 \pm 0.04 \mathrm{ng} \cdot \mathrm{L}^{-1}\right)$ for the sum of PAHs tested, which was also validated using a certified reference PAH mixture.

Keywords: Sensor; Electrochemical Impedance Spectroscopy; Self-assembled monolayers; Environmental pollutants; Surface engineering 


\section{Introduction}

Polycyclic aromatic hydrocarbons (PAHs) are a class of toxic, carcinogenic and mutagenic organic pollutants. They are present in the aquatic environment in the form of mixtures in trace concentrations due to their low water solubility [1-3]. More than 100 PAHs are known to occur naturally, but only 16 of them have been included by the US Environmental Protection Agency (EPA) as priority hazardous compounds [4], being $0.2 \mu \mathrm{g} \cdot \mathrm{L}^{-1}(\mathrm{ppb})$ the EPA's maximum contaminant level for these PAHs in drinking water [5].

Standard PAH analyses are mainly focused on bench-top instruments, including chromatographic, spectroscopic and immunoanalytical techniques [6-10]. These techniques usually require some pre-concentration and extraction procedures, hindering their integration for in-field analysis. Some electrochemical platforms, mainly based on voltammetric approaches, have shown to be a good alternative for the in situ PAH identification in aqueous samples [11-15]. Nonetheless, the use of electrochemical impedance spectroscopy (EIS) for PAHs determination is not often reported, except for a paper where a pyrene-based recognition platform is used for the selective sensing of pyrene [16].

Surface engineering is an indispensable step towards the achievement of a highly sensitive transducer. The covalent anchoring of ordered self-assembled monolayers (SAMs) onto solid supports is one of the most successful and facile routes to immobilize different nano- and macro-compounds to determine sensitively and selectively several analytes [17], [18]. In this regard, carbon nanotubes (CNTs) are an array of carbon hexagons with a $\pi$-electronic structure similar to that of a PAH molecule, promoting therefore abundant supramolecular $\pi$-interactions between PAHs 
and the external CNT surface. However, their use as recognition element beyond chromatographic approaches [19-23] still remains an unexplored field.

Inspired by the above facts, the objective of this work is studying the electrochemical performance of a CNT-based electrode as a highly sensitive impedimetric recognition platform for the direct ultra-low determination of the total amount of PAHs in water. For this goal, an Indium Tin Oxide (ITO) electrode has been covalently tuned with an amine-silane SAM carrying CNTs via amide bond formation. After optimization of the experimental preparation conditions, the present method has been validated using a standard material, demonstrating good sensitivity towards the impedimetric determination of this family of organic pollutants at part per trillion (ppt) levels even in complex samples, without needing any pretreatment step.

\section{Experimental}

\subsection{Apparatus}

Images of ITO substrates were acquired by Scanning Electron Microscopy (SEM), using a QUANTA FEI 200 FEG-ESEM with an acceleration voltage of $15 \mathrm{kV}$. Cyclic Voltammetry (CV) and EIS were performed on a Novocontrol Alpha-AN impedance analyzed with a potentiostat POT/GAL 30V/2A electrochemical interface using a conventional three-electrode configuration cell filled with $20 \mathrm{~mL}$ of a $0.1 \mathrm{M} \mathrm{KCl}$ solution containing $10 \mathrm{mM} \mathrm{K}_{3}\left[\mathrm{Fe}(\mathrm{CN})_{6} / \mathrm{K}_{4}\left[\mathrm{Fe}(\mathrm{CN})_{6}\right]\right.$ as a redox marker. The electrode configuration was: a single junction $\mathrm{Ag} / \mathrm{AgCl}$ (sat. $\mathrm{KCl}$ ) as reference electrode, a platinum wire as auxiliary electrode and the ITO-based substrates (exposed area: 6x10 $\mathrm{mm}^{2}$ ) as working electrodes. Cyclic voltammograms were carried out using a scan rate of $50 \mathrm{mV} \cdot \mathrm{s}^{-1}$. Impedimetric experiments were obtained using the following conditions: 
frequency range from $100 \mathrm{kHz}$ to $100 \mathrm{mHz}$; bias potential of $+150 \mathrm{mV}$ and $\mathrm{AC}$ amplitude of $5 \mathrm{mV}$. All the experiments were performed at room temperature and under environmental conditions. Charge transfer resistance $\left(R_{C T}\right)$ parameters were acquired by fitting the impedance spectra to the Randles equivalent circuit with $Z_{\text {view }}$ software (Scribner Associates Inc., USA).

\subsection{Chemical and reagents}

Single-walled carbon nanotubes (CNTs, $>95 \%$ of carbon purity, $<2 \mathrm{~nm}$ of outer diameter and 5-30 $\mu \mathrm{m}$ of length) were provided by SES Research (www.sesres.com). Activated-CNTs with either carboxylic groups (CNTs-COOH) or acyl groups (CNTsCOCl) were synthesized following references [24] and [25], respectively (see experimental part from the Supporting Information for further details). 3-aminopropyltrimethoxysilane (APTES, $\geq 98 \%$ ) and the 16 PAHs used in this study (pyrene (Pyr), naphthalene (Nap), anthracene (Ant), fluoranthene (Flt), benzo[a]pyrene (BaP), benzo[ghi]perylene (BghiP), coronene (Cor), acenaphthylene (Acy), benz[a]anthracene (BaA), benzo[b]fluoranthene $(\mathrm{B} b \mathrm{~F})$, benzo[k]fluoranthene $(\mathrm{B} k \mathrm{~F})$, fluorene (Fln), chrysene (Chry), dibenz[a,h]anthracene (DahA), indenol[1,2,3-cd]pyrene (InPy) and phenanthrene (Phe)) were purchased from Sigma-Aldrich (www.sigmaaldrich.com). ITO substrates were acquired from Delta Technologies (www.delta-technologies.com), as single-face-coated ITO over unpolished float glass with a resistance of $15-25 \Omega$. Aqueous solutions were prepared using deionized water from a Milli-Q system (Millipore). All organic solvents were of HPLC grade. Other chemical reagents were of the highest grade available and used without further purification.

An EPA 525 PAH Kit from Sigma-Aldrich was used as a certified reference material. The concentration of each compound in the acetonic mixture was $500 \mu \mathrm{g} \cdot \mathrm{L}^{-1}$. Stock solutions at the concentration of $5.0 \mu \mathrm{g} \cdot \mathrm{L}^{-1}$ were prepared in Milli-Q water by 
dilution. Aliquots of this stock solution were directly added into the electrochemical cell for calibration curve validation. Two complex mixture solutions were prepared by spiking $0.75 \mathrm{ng} \cdot \mathrm{L}^{-1}$ of 7 different PAH pollutants (Nap, Ant, Pyr, Flt, BaP, BghiP and Cor) in both Milli-Q and tap water, achieving a final $[\mathrm{PAHs}]_{\mathrm{TOTAL}}$ of $5.25 \mathrm{ng} \cdot \mathrm{L}^{-1}$.

\subsection{Electrode preparation}

Scheme 1 summarizes the general procedure of the surface modification. The electrode preparation was aimed at the covalent grafting of CNTs on the ITO-based substrates via three functionalization steps, which were adapted from reference [16]:

Step i) Activation of ITO substrates: ITO substrates (SI) of $90 \mathrm{~mm}^{3}\left(\mathbf{5} \mathrm{cm}^{2}\right)$ were first degreased in ultrasonic baths using a solvent series with increased polarity (dichloromethane, acetone and ethanol) for 5 min each and dried under a $\mathrm{N}_{2}$ steam. Afterwards, substrates were activated in an oxidizing bath of $\mathrm{NH}_{4} \mathrm{OH}: \mathrm{H}_{2} \mathrm{O}_{2}: \mathrm{H}_{2} \mathrm{O}$ (115) at $80{ }^{\circ} \mathrm{C}$ for $30 \mathrm{~min}$. Then, they were washed thoroughly with plenty of MilliQ water, rinsed with ethanol and dried under $\mathrm{N}_{2}$ steam, resulting in the activatedITO substrate (activated-SI).

Step ii) Amino-silane SAM formation: The activated-S1 substrates were quickly immersed in a $50 \mathrm{~mL}$ weighing bottle with grip stopper under $\mathrm{N}_{2}$ atmosphere containing $25 \mathrm{~mL}$ of a $0.5 \%$ (v/ v) APTES solution in dry toluene at $60{ }^{\circ} \mathrm{C}$ for $24 \mathrm{~h}$. Then, the substrates were cleaned with toluene and ethanol, obtaining $\mathbf{S}$.

Stage iii) Amide bond formation: Two different activated-CNTs (CNTs-COOH and CNTs- $\mathrm{COCl}$ ) were used for this approach using different synthetic conditions. For CNTs-COOH incorporation, a $0.1 \mathrm{mg} \cdot \mathrm{mL}^{-1}$ dispersion was used to promote the amide bond between them and the amino group from $\mathbf{S}$. For this aim, $\mathbf{S}$ substrates were firstly immersed in a $50 \mathrm{~mL}$ weighing bottle with grip stopper under $\mathrm{N}_{2}$ 
atmosphere filled with $25 \mathrm{~mL}$ of such dispersion doped with a 11 catalyst mixture of ED/ NHS for a specific time. For CNTs- $\mathrm{COCl}, \mathbf{S}$ substrates were also immersed in a $50 \mathrm{~mL}$ weighing bottle with grip stopper under $\mathrm{N}_{2}$ atmosphere filled with $25 \mathrm{~mL}$ of a $0.1 \mathrm{mg} \cdot \mathrm{mL}^{-1}$ dispersion containing trimethylamine $(\mathrm{DO} \mu \mathrm{L})$ as catalyst in order to promote the amide bond formation. Finaly, the substrates were rinsed with plenty of toluene and ethanol and finaly dried with $\mathrm{N}_{2}$, resulting in $\mathbf{S}$. The different experimental synthetic conditions (solvent, incubation time and reactive group) are summarized in Table 1

Scheme 1. Schematic representation of the three functionalization stages for the functionalization of the ITO electrode with CNTs as recognition agents: i) ITO activation with hydroxyl groups, ii) APTES-based SAM formation and iii) subsequently grafting of activated-CNTs via amide bond formation.

\subsection{Electroanalytical assay}

All the electroanalytical experiments were carried out impedimetrically using the aforementioned experimental conditions. Firstly, Pyr was used as the PAH proof. Its determination was recorded by adding directly different aliquots of Pyr into the electrochemical cell, where a 10-minute incubation step took place under stirring conditions in order to promote supramolecular $\pi$-interactions between the recognition agent and the PAH analyte. A freshly saturated stock solution of Pyr (135 ppb) was prepared in Milli-Q water without any further treatment. Different dilutions from the saturated stock solution were necessary for aliquots addition. The ame methodology was employed for the rest of PAH analytes used (Nap, Ant, BaP and BghiP). Table S2 summarizes the experimental details employed for PAHs determination at the electrochemical cell. Both reference and spiked mixture solutions were analyzed by 
adding directly an aliquot into the electrochemical cell. Each experiment was repeated three times and measurements were acquired per triplicate in order to observe both reproducibility and repeatability $(n=9)$.

\section{Results and Discussion}

\subsection{Choice of materials}

Single-walled CNTs have been chosen as the recognition element since they have demonstrated better adsorption affinity for several PAHs rather than other common carbon nanomaterials [26]. However, their use for the electrochemical PAH determination had not been exploited before. Additionally, since a previous activation of the carbon surface with oxidative groups is mandatory for the amide bond formation on the SAM-based electrode surface, CNTs present better electrochemical performance than graphene oxide (the activated form of graphene, which is insulating). ITO has been employed as the transducer platform because it can be easily functionalized via covalently anchoring with organic films. The monolayer produced by self-assembly gives tremendous versatility and has been shown to be very promising in different disciplines, including biosensing and organic electronics [17], [27]. Accordingly, considering the synergistic $\pi$-interactions reported between single-walled CNTs and PAHs, and taking into account that EIS is a much more sensitive electrochemical tool than conventional voltammetric methods, the present assay is expected to monitor small impedimetric changes on the CNT-based electrode surface after interacting with low PAHs concentrations.

\subsection{Engineering and characterization of the electrochemical recognition platforms}

The experimental conditions employed in the last surface functionalization step (step

iii) in Scheme 1) are crucial to ensure the successful integration of CNTs, and have 
therefore, a strong impact on the electroanalytical performance of the recognition sensing platform S3. Thus, several experimental conditions such as solvent, incubation time or reactive groups, were interrogated in order to optimize the device response. The S3 surfaces were fully electrochemically characterized by means of CV and EIS. All electrochemical data are collected in Table 1. Further surface characterization data, such as water contact angle and X-ray Photoelectron Spectroscopy (XPS) measurements is available in Figure S1 and Table S1, respectively.

For the electrochemical characterization, the $\left[\mathrm{Fe}(\mathrm{CN})_{6}\right]^{3-/ 4-}$ was employed as the redox marker since it is a commonly used redox couple to investigate the electrochemical performance of modified electrode surfaces. CV provides information about the accessibility and reversibility of the redox marker to be oxidized or reduced at the electrode surface by analyzing the current intensity peaks $\left(I_{p}\right)$ and the redox peaks separation $(\Delta E)$. As it is summarized in Table 1, the formation of the APTES-based SAM (from S1 to S2, step ii)) was confirmed since an $I_{p}$ decrease accompanied by a $\Delta E$ increase was observed, indicating a lower electron transfer reversibility. This fact is attributed to the insulating properties of the APTES monolayer. The electronic transfer capabilities in the frequency domain were also studied by EIS, where the charge transfer resistance $\left(\mathrm{R}_{\mathrm{CT}}\right)$ values were determined by fitting the Nyquist plots (imaginary impedance vs. real impedance) with a typical Randles equivalent circuit [28]. As it was expected from the CV data, a $\mathrm{R}_{\mathrm{CT}}$ increase was observed after APTES-based SAM formation owing to the electrode surface is blocked, obstructing the interfacial redox reaction between the electrode and the probe in the electrolyte solution. 
Table 1. Summary of the synthetic conditions and electrochemical characterization data at each functionalization stage.

All the S3 surfaces formed after step iii) using different experimental parameters were characterized. It is known that CNTs present more chemical active points at the edges and at the defects of their walls [29]. Therefore, the dispersibility, the amount of CNTs and/or the nature of the functional groups are key parameters to optimize in order to achieve an optimum electrochemical performance. From Table $\mathbf{1}$ it is possible to observe small electrochemical changes from $\mathbf{S 2}$ to $\mathbf{S 3 - A}$, fact that can be attributed to an unsuccessful covalently grafting of CNTs on the electrode surface when using DMSO as solvent. Nonetheless, this was solved by changing the solvent for DMF (S3$\mathbf{B}, \mathbf{C}$, and $\mathbf{D})$. Comparing these three surfaces, the $\mathbf{S} 3-\mathbf{B}$ showed the highest $\Delta E$ and $\mathrm{R}_{\mathrm{CT}}$ and the lowest $I_{p}$. These results are ascribed to the role of oxygen-containing groups on the electrochemistry of CNTs that lead to slower heterogeneous electron-transfer for $\left[\mathrm{Fe}(\mathrm{CN})_{6}\right]^{3-/ 4-}[30]$. Therefore, the electrochemical performance was obviously improved exchanging the carboxylic groups (S3-B) for the acyl chlorine groups (S3-C and S3D). Finally, the better electrochemical parameters (lowest $\Delta E$ and $\mathrm{R}_{\mathrm{CT}}$ and higher $I_{p}$ ) obtained for the S3-D are due to a higher amount of CNTs derived from increasing the incubation time from $24 \mathrm{~h}$ to $96 \mathrm{~h}$. In order to support this explanation, morphological studies were carried out to gain qualitative information about the morphology, distribution and covering of CNTs throughout the S3-based electrode surfaces.

\subsection{Scanning Electronic Microscope Images}

Figure 1 shows some representative SEM images obtained for the S3-based surfaces. Comparing those surfaces functionalized with CNTs-COOH (S3-A vs. S3-B), a much higher coverage for S3-B was found since very few CNTs were observed in S3-A, 
indicating that DMF is the most efficient solvent for CNTs incorporation. These results are in concordance with electrochemical results, where no significant changes between S2 and S3-A were observed because of the low CNT coverage (see Table 1). Regarding the influence of the nature of the activated functional groups (S3-B vs. S3C), similar coverage was observed for both substrates. Nonetheless, S3-B showed a higher agglomeration of CNTs owing to the poor dispersibility of CNTs-COOH in DMSO (see Figure S2). Finally, if S3-C and S3-D surfaces are contrasted, a coverage enhancement was obtained in S3-D due to the longer incubation time (24 h vs. 96 h) employed.

\section{Figure 1}

\subsection{Electroanalytical performance}

After characterizing the four $\mathbf{S 3}$ substrates, the next step consisted in determining the most suitable s3-based recognition platform for PAHs determination. The electroanalytical assay is focused on the excellent supramolecular $\pi$-interactions established between the CNTs attached on the electrode surface and the aromatic planar structure of the PAH analytes.

Firstly, impedimetric measurements were undertaken to monitor Pyr as a proof PAH analyte, using Nyquist plots. Different aliquots of the analyte were directly added in the electrochemical cell and aged for 10 min under stirring conditions. Initially, the electrochemical response of the bare $\mathbf{S 1}$ electrode (reference test) was explored, where no changes towards $[\mathrm{Pyr}]$ were observed (see Figure 2A). This fact indicates that the bare ITO electrode is not capable to recognize Pyr molecules. Subsequently, S3 
substrates were studied (see Figure S3). From an electrochemical point of view, it is expected that the formation of supramolecular interactions between CNT and Pyr would generate an increase in the $\mathrm{R}_{\mathrm{CT}}$ parameter [28].

Figure 2B depicts the calibration curves towards [Pyr] corresponding to each S3 recognition platform. In order to compare the results obtained from the different electrodes used, and to obtain independent and reproducible results, the relative $\Delta \mathrm{R}^{\prime}{ }_{\mathrm{CT}}$ signal $\left(\Delta R_{C T} / R_{0}\right)$ was used, where $R_{0}$ is the electron transfer resistance of the electrode at the initial state and $\Delta \mathrm{R}_{\mathrm{CT}}$ is the difference between the electron transfer resistance value measured after incubation with PAHs and $\mathrm{R}_{0}$ [16]. As it was expected from the SEM characterization, no impedimetric changes with increasing [Pyr] where observed for those substrates obtained using DMSO as the solvent (S3-A) because of the lack of CNTs on the electrode surface. Nonetheless, a significant electroanalytical response was found changing the solvent for DMF (S3-B), yielding a detection limit as low as 1.75 ng $\cdot \mathrm{L}^{-1}$. Afterwards, the S3-B and S3-C electrode responses were compared, which, as mentioned, were prepared using CNTs with two different activation groups (CNTs$\mathrm{COOH}$ and $\mathrm{CNTs}-\mathrm{COCl}$, respectively). As it is possible to observe from Figure 2 B, equal detection limits and very similar sensitivities towards Pyr were found independently of the reactive group used. However, it is important to point out that solutions containing CNTs-COCl presented much better dispersibility (see Figure S2), fact that increased the reproducibility of the system (note lower error bars for S3-C). The incubation time was also studied. Comparing S3-C and S3-D substrates, a significant improvement in sensitivity (65.7\%) was obtained after increasing the incubation time from $24 \mathrm{~h}$ to $96 \mathrm{~h}$. Hence, and in concordance with electrochemical and morphological results, the most suitable electroanalytical platform for PAHs determination is the S3-D. 
The present approach was also compared with a drop-casted ITO electrode, which was prepared by casting $200 \mu \mathrm{L}$ of a $0.1 \mathrm{mg} \cdot \mathrm{mL}^{-1} \mathrm{CNT}-\mathrm{COCl}$ solution in DMF, where no significant response towards different [Pyr] was observed (see Figure S4). Accordingly, it is possible to conclude at this point that surface engineering and realizing an optimized experimental protocol for the electrode preparation are indispensable steps for the achievement of a highly sensitive and reproducible CNTbased electrochemical recognition platform capable to impedimetrically respond to small surface changes.

\section{Figure 2}

\subsection{Determination of PAHs in water and applications}

The feasibility of the optimized S3-D recognition platform towards the electrochemical determination of total PAHs in water was explored. A multi-calibrate curve (see Figure 3, curve a) was performed increasing the total concentration of PAHs by adding aliquots of different pollutants with increasing number of benzenic rings $(\mathrm{m})$ according to: Nap, Ant, $\mathrm{B} a \mathrm{P}$ and $\mathrm{B} g h i \mathrm{P}$, with $m=2,3,5$ and 6, respectively. This protocol was carried out in order to avoid the dependence of the PAH nature. Good linearity was observed over the concentration range of $1.75-7.00 \mathrm{ng} \cdot \mathrm{L}^{-1}$, with a correlation coefficient $\left(\mathrm{r}^{2}\right)$ of 0.9997 and a limit of detection (LOD) of $1.75 \pm 0.04 \mathrm{ng} \cdot \mathrm{L}^{-}$

${ }^{1}$ for sum of PAHs. Interestingly, the present impedimetric recognition platform does not differentiate between Pyr and other PAHs (Figure 3, curve a vs. curve b), indicating that the device exhibits a universal response for such PAHs and that Pyr can act as a standard PAH for calibration curve construction. 
A series of certificated standard solutions (EPA 610-N PAH kit from SigmaAldrich) containing each of the PAHs at two concentration levels of 2.0 and $5.5 \mathrm{ng} \cdot \mathrm{L}^{-1}$ were prepared for the validation of the calibration curve. For each level, three replicates and determinations ( $n=9$ ) were performed using the optimized electrochemical device. After interpolating the average impedimetric response on the Pyr calibration curve, the recoveries of the method were in the range from $93.8 \pm 0.5 \%$ to $100.7 \pm 1.4 \%$ for concentrations levels of 2.0 and $5.5 \mathrm{ng} \cdot \mathrm{L}^{-1}$, respectively (see Figure S5). These results suggest that this method is very reproducible and can be suitable for the determination of the priority EPA’s PAH pollutants in aqueous samples.

In order to evaluate the applicability of the developed method, two complex mixture solutions were tested by determining the PAHs in spiked water samples using both Mill-Q water and tap water. Spiked water samples includes 7 different EPA pollutants $\left([\mathrm{Nap}]=[\mathrm{Ant}]=[\mathrm{Pyr}]=[\right.$ Flt $\left.]=[\mathrm{BaP}]=[\mathrm{BghiP}]=[\mathrm{Cor}]=0.75 \mathrm{ng} \cdot \mathrm{L}^{-1}\right)$, yielding a $[\mathrm{PAHs}]_{\text {TOTAL }}$ of $5.25 \mathrm{ng} \cdot \mathrm{L}^{-1}$. Interpolating the average impedimetric response on the Pyr calibration curve, a superb recovering as good as $97.8 \pm 4.6 \%$ and $98.5 \pm$ 5.9\% was achieved for the Milli-Q water and tap water samples, respectively. Thus, it is important to point out that tap water samples do not present a matrix effect and therefore, do not interfere with the assay. These results clearly demonstrate excellent merits of the optimized CNT-based recognition platform (S3-D) towards the screening of sum of PAHs at part per trillion levels $(\sim \mathrm{pM})$ even in complex matrices.

\section{Figure 3}

\subsection{Comparison of the method with other works}


The developed impedimetric assay has been compared with other previous methods that were used for the determination of PAHs in aqueous samples using carbon-based materials as recognition platforms. Table 2 summarizes several figures of merits of some analytical methods as well as the ones found this work. According to Table 2, it can be concluded that the present CNT-based EIS recognition platform exhibits simplicity, rapidity, reproducibility and ease of operation, with the added value that no further pretreatments are needed to achieve the ultra-low PAH concentrations demanded by the EPA. Even though, further experimentation is needed to validate these important results in real environmental samples since it has been demonstrated, for example, the sorption of some PAHs to humic acids [31].

Table 2. Method comparisons for analysis of PAHs in water.

\section{Conclusions}

A CNT-functionalized electrode has been validated for the simple, rapid and sensitive electrochemical determination of total PAHs pollutants in water at part per trillion levels via supramolecular $\pi$-interactions. It has been demonstrated that surface engineering is an indispensable step towards the achievement of a highly sensitive and reproducible CNT-based impedimetric recognition platform, pointing out the key role played by the APTES-based SAM since no response was observed when CNTs where drop-casted on an ITO surface. The present electrochemical assay is straightforward and faster when compared with conventional analysis since does not require expensive instrumentation, further pretreatments and/or large sample volumes, and can be easily miniaturized owing to the transduction method is electronic. The utility of this impedimetric method for the screening of total PAHs is shown by conducting a proof- 
of-concept field demonstration, using both standard solutions and spiked complex mixtures, being expected to be very useful for sensing approaches in real environmental samples. However, a more systematic interference study is needed to validate the method, and is the object of our ongoing research. Hence, this work opens up novel approaches for the development of novel 'at-point-of-use’ sensors for monitoring in situ pollutant carbon-based nanomaterials in aqueous samples at ultra-trace levels.

\section{Acknowledgments}

This work was funded by the ERC StG 2012-306826 e-GAMES. The authors also thank the DGI (Spain) project FANCY CTQ2016-80030-R, the Generalitat de Catalunya (2017-SGR-918) and the Spanish Ministry of Economy and Competitiveness, through the "Severo Ochoa” Programme for Centers of Excellence in R\&D (SEV-20150496). Dr. J. Muñoz gratefully acknowledges the “Juan de la Cierva” programme.

\section{References}

1. Abdel-Shafy HI, Mansour MS (2016) A review on polycyclic aromatic hydrocarbons: source, environmental impact, effect on human health and remediation. Egypt J Pet 25:107-123.

2. IARC Working Group on the Evaluation of Carcinogenic Risks to Humans (2010) Some non-heterocyclic polycyclic aromatic hydrocarbons and some related exposures, IARC monographs on the evaluation of carcinogenic risks to humans. 92:1. 
3. Kim K-H, Jahan SA, Kabir E, Brown RJ (2013) A review of airborne polycyclic aromatic hydrocarbons (PAHs) and their human health effects. Environ Int 60:7180.

4. Bansal V, Kumar P, Kwon EE, Kim K-H (2017) Review of the quantification techniques for polycyclic aromatic hydrocarbons (PAHs) in food products. Crit Rev Food Sci Nutr 57(15):3297-3312.

5. Bruzzoniti MC, Fungi M, Sarzanini C (2010) Determination of EPA's priority pollutant polycyclic aromatic hydrocarbons in drinking waters by solid phase extraction-HPLC. Anal Methods 2:739-745.

6. Saini SS, Kabir A, Rao ALJ, Malik AK, Furton KG (2017) A Novel Protocol to Monitor Trace Levels of Selected Polycyclic Aromatic Hydrocarbons in Environmental Water Using Fabric Phase Sorptive Extraction Followed by High Performance Liquid Chromatography-Fluorescence Detection. Separations 4(2):22.

7. Poster DL, Schantz MM, Sander LC, Wise SA (2006) Analysis of polycyclic aromatic hydrocarbons (PAHs) in environmental samples: a critical review of gas chromatographic (GC) methods. Anal Bioanal Chem 386:859-881.

8. Andrews AB, Wang D, Marzec KM, Mullins OC, Crozier KB (2015) Surface enhanced Raman spectroscopy of polycyclic aromatic hydrocarbons and molecular asphaltenes. Chem Phys Lett 620:139-143.

9. Li X, Kaattari SL, Vogelbein MA, Vadas GG, Unger MA (2016) A highly sensitive monoclonal antibody based biosensor for quantifying 3-5 ring polycyclic aromatic hydrocarbons (PAHs) in aqueous environmental samples. Sens Biosensing Res $7: 115-120$ 
10. Du J, Xu J, Sun Z, Jing C (2016) Au nanoparticles grafted on Fe3O4 as effective SERS substrates for label-free detection of the 16 EPA priority polycyclic aromatic hydrocarbons. Anal Chim Acta 915:81-89.

11. Lux G, Langer A, Pschenitza M, Karsunke X, Strasser R, Niessner R, Knopp D, Rant U (2015) Detection of the carcinogenic water pollutant benzo[a]pyrene with an electro-switchable biosurface. Anal Chem 87(8):4538-4545.

12. Lin Y-Y, Liu G, Wai CM, Lin Y (2007) Magnetic beads-based bioelectrochemical immunoassay of polycyclic aromatic hydrocarbons. Electochem Commun 9(7):1547-1552.

13. Ni Y, Wang P, Song H, Lin X, Kokot S (2014) Electrochemical detection of benzo(a)pyrene and related DNA damage using DNA/hemin/nafion-graphene biosensor. Anal Chim Acta 821:34-40.

14. Cho H-H, Smith BA, Wnuk JD, Fairbrother DH, Ball WP (2008) Influence of surface oxides on the adsorption of naphthalene onto multiwalled carbon nanotubes. Environ Sci Technol 42:2899-2905.

15. Del Carlo M, Di Marcello M, Perugini M, Ponzielli V, Sergi M, Mascini M, Compagnone D (2008) Electrocehmical DNA biosensor for polycyclic aromatic hydrocarbons detection. Microchim Acta 163(3-4):163-169.

16. Muñoz J, Crivillers N, Mas-Torrent M (2017) Carbon-rich monolayers on ITO as highly sensitive platforms for detecting polycyclic aromatic hydrocarbons in water: The case of pyrene. Chem Eur J 23(61):15289-15293.

17. Casalini S, Bortolotti CA, Leonardi F, Biscarini F (2017) Self-assembled monolayers in organic electronics. Chem Soc Rev 46(1):40-71.

18. Chaki NK, Vijayamohanan K (2002) Self-assembled monolayers as a tunable platform for biosensors applications. Biosens Bioelectron 17(1-2):1-12 
19. Pan B, Xing B (2008) Adsorption mechanisms of organic chemicals on carbon nanotubes. Environ Sci Technol 42(24):9005-9013.

20. Leyton P, Gómez-Jeria J, Sanchez-Cortes S, Domingo C, Campos-Vallette M (2006) Carbon nanotube bundles as molecular assemblies for the detection of polycyclic aromatic hydrocarbons: surface-enhanced resonance Raman spectroscopy and theoretical studies. J Phys Chem B 110:6470-6474.

21. Ma J, Xiao R, Li J, Yu J, Zhang Y, Chen L (2010) Determination of 16 polycyclic aromatic hydrocarbons in environmental water samples by solid-phase extraction using multi-walled carbon nanotubes as adsorbent coupled with gas chromatography-mass spectrometry. J Chromatogr A 1217:5462-5469.

22. Shen M, Xia X, Wang F, Zhang P, Zhao X (2012) Influences of multiwalled carbon nanotubes and plant residue chars on bioaccumulation of polycyclic aromatic hydrocarbons by Chironomus plumosus larvae in sediment. Environ Toxicol Chem 31:202-209.

23. Thiruppathi M, Thiyagarajan N, Gopinathan M, Zen J-M (2016) Role of defect sites and oxygen functionalities on preanodized screen printed carbon electrode for adsorption and oxidation of polyaromatic hydrocarbons. Electrochem Commun 69:15-18.

24. Muñoz J, Cespedes F, Baeza M (2015) Effect of carbon nanotubes purification on electroanalytical response of near-percolation amperometric nanocomposite sensors. J Electrochem Soc 162:B217-B224.

25. Cabana L, González-Campo A, Ke X, Van Tendeloo G, Núñez R, Tobias G (2015) Efficient Chemical Modification of Carbon Nanotubes with Metallacarboranes. Chem Eur J 21:16792-16795. 
26. Yang K, Zhu L, Xing B (2006) Adsorption of polycyclic aromatic hydrocarbons by carbon nanomaterials. Environ Sci Technol 40(6):1855-186.

27. Marchante E, Crivillers N, Buhl M, Veciana J, Mas-Torrent M (2016) An Electrically Driven and Readable Molecular Monolayer Switch Based on a Solid Electrolyte. Angew Chem Int Ed 128(1):376-380.

28. Muñoz J, Montes R, Baeza M (2017) Trends in Electrochemical Impedance Spectroscopy involving nanocomposite transducers: characterization, architecture surface and bio-sensing. Trends Anal Chem 97:201-215.

29. Pumera M (2009) The electrochemistry of carbon nanotubes: fundamentals and applications. Chem Eur J 15(20):4970-4978.

30. Pumera M (2007) Electrochemical properties of double wall carbon nanotube electrodes. Nanoscale Res Lett 2(2):87.

31. Nielsen T, Siigur K, Helweg C, Jørgensen O, Hansen PE, Kirso U (1997) Sorption of polycyclic aromatic compounds to humic acid as studied by high-performance liquid chromatography. Environ Sci Technol 31 (4):1102-1108.

32. Rajabi M, Moghadam AG, Barfi B, Asghari A (2016) Air-assisted dispersive microsolid phase extraction of polycyclic aromatic hydrocarbons using a magnetic graphitic carbon nitride nanocomposite. Microchim Acta 183 (4):1449-1458.

33. 33. Tiu BDB, Krupadam RJ, Advincula RC (2016) Pyrene-imprinted polythiophene sensors for detection of polycyclic aromatic hydrocarbons. Sensors and Actuators B: Chemical 228:693-701.

34. Wang W, Ma R, Wu Q, Wang C, Wang Z (2013) Magnetic microsphere-confined graphene for the extraction of polycyclic aromatic hydrocarbons from environmental water samples coupled with high performance liquid chromatography-fluorescence analysis. J Chromatogr A 1293:20-27. 
35. Menezes HC, de Barcelos SMR, Macedo DFD, Purceno AD, Machado BF, Teixeira APC, Lago RM, Serp P, Cardeal ZL (2015) Magnetic N-doped carbon nanotubes: a versatile and efficient material for the determination of polycyclic aromatic hydrocarbons in environmental water samples. Anal Chim Acta 873:51-56.

36. Han Q, Wang Z, Xia J, Chen S, Zhang X, Ding M (2012) Facile and tunable fabrication of Fe3O4/graphene oxide nanocomposites and their application in the magnetic solid-phase extraction of polycyclic aromatic hydrocarbons from environmental water samples. Talanta 101:388-395.

37. Wang W-D, Huang Y-M, Shu W-Q, Cao J (2007) Multiwalled carbon nanotubes as adsorbents of solid-phase extraction for determination of polycyclic aromatic hydrocarbons in environmental waters coupled with high-performance liquid chromatography. J Chromatogr A 1173 (1-2):27-36.

38. Fähnrich K, Pravda M, Guilbault G (2003) Disposable amperometric immunosensor for the detection of polycyclic aromatic hydrocarbons (PAHs) using screen-printed electrodes. Biosens Bioelectron 18 (1):73-82.

\section{Supplementary Material}

Supplementary material for this article is given via link at the end of the document. 


\section{Figure Captions}

Figure 1. SEM images obtained for the four different S3-based recognition platforms: A) S3-A (inset: S1 blank) and B) S3-B, C) S3-C and D) S3-D with higher magnification insets.

Figure 2. A) Electroanalytical response at the bare $\mathbf{S 1}$ electrode (reference test) in the presence of increasing concentrations of Pyr (0 to $\left.7.00 \mathrm{ng} \cdot \mathrm{L}^{-1}\right)$. Inset: molecular Pyr structure. B) Calibration curve representing the $\Delta \mathrm{R}_{\mathrm{CT}}{ }_{\mathrm{cs}}$. [Pyr] at the four different S3based recognition platforms with their corresponding error bars $(n=9)$. Measurements were obtained after 10 min incubation with the Pyr aliquots.

Figure 3. Calibration curve representing the $\Delta \mathrm{R}^{\prime}{ }_{\mathrm{CT}} v s$. [PAHs] adding different aliquots of PAHs with increasing the number of aromatic rings ( $m$ : Nap $<$ Ant $<\mathrm{B} a \mathrm{P}<\mathrm{BghiP}$ ) at the S3-D recognition platform (curve a), which is compared to the calibration curve obtained by [Pyr] (curve b). Calibration plots are represented with their corresponding error bars $(n=9)$. Insets: molecular PAHs structures tested. 